\title{
ON THE ASYMPTOTIC DISTRIBUTION OF EIGENVALUES OF SOME NONSELFADJOINT PROBLEMS
}

\author{
PETER HESS
}

\section{Introduction and statement of the results}

In [4] we studied the question of the location of the spectrum of the linear elliptic eigenvalue problem

$$
\mathscr{L} u=\lambda m u \text { in } \Omega, \quad u=0 \text { on } \partial \Omega,
$$

where $m \in L^{\infty}(\Omega)$ is a real-valued (possibly indefinite) weight function, $m \neq \equiv 0$. We proved in particular that if the top-order coefficients of $\mathscr{L}$ are real-valued and smooth, and if $m \geqslant 0$ in the bounded domain $\Omega$, then (1) has a discrete spectrum and the eigenvalues condense along the positive axis: for arbitrary $\varepsilon$ with $0<\varepsilon<\pi / 2$ all the eigenvalues $\lambda$, except possibly a finite number of them, lie in the sector $G_{\varepsilon}:=\{\zeta \in \mathbb{C}:|\arg \zeta|<\varepsilon\}$. The generalized eigenfunctions are relatively complete, in a sense made precise there. Here we consider the question of the asymptotic distribution of the eigenvalues. For simplicity we assume $\mathscr{L}$ to be of second order with real-valued coefficient functions; it is however straightforward to extend the results to higher-order problems and/or complex-valued lower order coefficients. let $\mathscr{L}$ :

Let $\Omega \subset \mathbb{R}^{N}(N \geqq 1)$ be a bounded domain having smooth boundary $\partial \Omega$, and

$$
\mathscr{L} u=-\sum_{j, k=1}^{N} a_{j k} \frac{\partial^{2} u}{\partial x_{j} \partial x_{k}}+\sum_{k=1}^{N} a_{k} \frac{\partial u}{\partial x_{k}}+a_{0} u
$$

be a strongly uniformly elliptic differential expression with real-valued coefficient functions $a_{j k}=a_{k j} \in C^{1}(\bar{\Omega}), a_{k}, a_{0} \in L^{\infty}(\Omega), a_{0} \geqq 0$. Further, let $m \in L^{\infty}(\Omega)$ be a weight function with $m \geqq 0, m \neq 0$. Setting $\Omega_{+}:=\{x \in \Omega: m(x)>0\}$, we assume that meas $\left(\Omega_{+} \backslash\right.$ int $\left.\Omega_{+}\right)=0$ (which holds, for example, if $m$ is continuous). For $t \geqq 0$ let $n(t)$ denote the number of eigenvalues $\lambda$ of problem (1) with $\operatorname{Re} \lambda \leqq t$.

THEOREM 1. $n(t) \sim c t^{\frac{1}{2} N}$ as $t \rightarrow+\infty$, where $c=\int_{\Omega} m(x)^{\frac{1}{2} N} \mu_{\mathscr{L}_{0}}(x) d x$ and

$$
\mu_{\mathscr{L}_{0}}(x):=(2 \pi)^{-N} \int_{\left\{\xi \in \mathbb{R}^{N}: \Sigma a_{j k}(x) \xi_{j} \xi_{k}<1\right\}} d \xi .
$$

This result is well-known in the standard situation $m=1$ (e.g. Agmon [1]). In the present generality it is obtained by considering $\mathscr{L}$ as a lower order perturbation of the formally selfadjoint differential expression

$$
\mathscr{L}_{0}:=-\sum_{j, k=1}^{N} \frac{\partial}{\partial x_{j}}\left(a_{j k} \frac{\partial}{\partial x_{k}}\right),
$$

Received 1 September 1985.

1980 Mathematics Subject Classification 35P20.

Bull. London Math. Soc. 18 (1986) 181-184 
applying the results of e.g. Pleijel [6] or Fleckinger and Lapidus [2] on the asymptotic distribution of the eigenvalues of the variational problem

$$
\mathscr{L}_{0} u=\lambda m u \text { in } \Omega, \quad u=0 \text { on } \partial \Omega,
$$

and using an abstract perturbation result which should be of independent interest.

Let $\mathscr{H}$ be an (infinite-dimensional) separable Hilbert space over $\mathbb{C}$, and for $0<p \leqq \infty$ let $C_{p}$ denote the two-sided ideal in $\mathscr{L}(\mathscr{H})$ consisting of the (compact) operators $A$ for which the eigenvalues of $\left(A^{*} A\right)^{\frac{1}{2}}$, arranged in decreasing order and repeated according to multiplicity, form an $l_{p}$-sequence. We say that $\lambda \in \mathbb{C}$ is a characteristic value of $A$ if there is an $x \neq 0$ such that $x=\lambda A x$; of course $\lambda \neq 0$, and $x$ is an eigenfunction of $A$ corresponding to the eigenvalue $\lambda^{-1}$. We denote the range and null space of $A$ by $R(A)$ and $N(A)$ respectively.

THEOREM 2. Let $A:=H(I+S)$, where $H \in \mathscr{L}(\mathscr{H})$ is compact, selfadjoint, nonnegative (that is, $(H x, x) \geqq 0 \forall x \in \mathscr{H})$ and belongs to the class $C_{p}$ for some $p<\infty$, while $S \in \mathscr{L}(\mathscr{H})$ is compact and such that $(I+S)$ is invertible.

Let $\mathscr{H}$ be orthogonally decomposed as $\mathscr{H}=\mathscr{H}_{1} \oplus \mathscr{H}_{2}$, where $\mathscr{H}_{1}=\overline{R(H)}$ and $\mathscr{H}_{2}=\mathcal{N}(\mathscr{H})$, and suppose that

$$
\mathscr{H}_{1} \cap(I+S)^{-1} \mathscr{H}_{2}=\{0\} .
$$

If there exists a nondecreasing function $\phi$ on $\mathbb{R}^{+}$with $\phi(t) \rightarrow+\infty$ as $t \rightarrow+\infty$, satisfying

(2) $\frac{\phi(s)}{\phi(t)} \leqq\left(\frac{s}{t}\right)^{\gamma}$ for all sufficiently large $t<s$ and some constant $\gamma$ with $0<\gamma<p$,

and such that $\lim _{t \rightarrow+\infty} n(t, H) / \phi(t)=1$, then

$$
\lim _{t \rightarrow+\infty} n(t, A) / n(t, H)=1 .
$$

Here $n(t, A)$ and $n(t, H)$ denote the distribution functions of the characteristic values of $A$ and $H$, respectively (or since by [4, Theorem 1] almost all characteristic values of $A$ lie in the sector $G_{\varepsilon}, n(t, A)$ is the number of characteristic values $\lambda$ of $A$ with $\operatorname{Re} \lambda \leqq t$ ). Theorem 2 extends an assertion of Keldyš [5] (compare [3, Theorem 11.1]) to the case where $H$ may have nontrivial nullspace, and relies on the results of [4]. Condition (2) is trivially satisfied for $\phi(t)=c t^{\gamma}(c>0)$.

Remarks. 1. It is not clear whether Theorems 1 and 2 can also be obtained by a limiting procedure, looking first at definite problems, since in the nonselfadjoint case there are no monotonicity arguments available (compare with $[\mathbf{2}$, proof of Theorem 3.1]).

2. The results on the variational eigenvalue problem $\left(1_{0}\right)$ in $[2,6$, as well as the perturbation result in [4], hold for weight functions $m \in L^{\infty}(\Omega)$ which may change sign. We have, however, not been able to prove that the spectrum of $(1)$ and $\left(1_{0}\right)$ still have the same asymptotic distribution in the case when $m$ is indefinite rather than semidefinite.

3. There are various recent results on perturbations of (abstract) selfadjoint operators preserving the asymptotic properties of the spectrum (for example, Ramm [7]). These results do not apply here since the perturbed mappings are not normal. 


\section{Proofs}

Once Theorem 2 is proved, the assertion of Theorem 1 follows immediately along the lines of Hess [4, proof of Theorem 2], using the results of $[2,6]$. We therefore do not reproduce it.

The proof of Theorem 2 parallels that of [3, Theorem 11.1]; we give only the details of those steps which differ. We start with the following auxiliary result which is a variant of the Lemma in [4]:

LEMMA 1. Let $H \in \mathscr{L}(\mathscr{H})$ be compact, selfadjoint and nonnegative, and let $T \in \mathscr{L}(\mathscr{H})$ be compact. Decompose $\mathscr{H}=\mathscr{H}_{1} \oplus \mathscr{H}_{2}$, where $\mathscr{H}_{1}=\overline{R(H)}, \mathscr{H}_{2}=N(H)$. For $0<\varepsilon<\pi$ let $F_{\varepsilon}:=\{\zeta \in \mathbb{C}: \varepsilon \leqq \arg \zeta \leqq 2 \pi-\varepsilon\}$. Then

$$
\lim _{\substack{\lambda \in F_{\varepsilon} \\|\lambda| \rightarrow \infty}}\left\|\left.T(I-\lambda H)^{-1}\right|_{\mathscr{H}_{1}}\right\|=0 .
$$

To $S \in \mathscr{L}(\mathscr{H})$ as given in Theorem 2, we associate the (compact) operator $T$ such that $I-T=(I+S)^{-1}$. Fix $\varepsilon$ with $0<\varepsilon<\pi$. For $\lambda \in F_{\varepsilon}$ the factorization

$$
I-\lambda A=\left(I-T(I-\lambda H)^{-1}\right)(I-\lambda H)(I+S)
$$

holds. The following assertions are proved in [4]:

(i) $I-\lambda A$ is injective for $\lambda \in F_{\varepsilon, R}:=\left\{\zeta \in F_{\varepsilon}:|\zeta| \geqq R\right\}$, provided $R$ is sufficiently large;

(ii) $\left(I-T(I-\lambda H)^{-1}\right)^{-1}$ is bounded on $F_{\varepsilon, R}$;

(iii) $\mathscr{H}_{1}$ is invariant under $H$ and $A$ and equals the closed linear hull of all the generalized eigenvectors of $A$ belonging to characteristic values $\lambda(\neq \infty)$.

For $\lambda \in F_{\varepsilon, R}$ let $A(\lambda):=\lambda^{-1}\left((I-\lambda A)^{-1}-I\right)=A(I-\lambda A)^{-1}$ denote the Fredholm resolvent. As in $[3$, p. 279] we have

and thus

$$
A(\lambda)-H(\lambda)=A(\lambda) T(I-\lambda H)^{-1}
$$

where

$$
A(\lambda)=H(\lambda)(I+C(\lambda)),
$$

$$
C(\lambda)=\left(I-T(I-\lambda H)^{-1}\right)^{-1}-I \text {. }
$$

LeMma 2. $\left\|\left.C(\lambda)\right|_{\mathscr{H}_{1}}\right\| \rightarrow 0$ as $\lambda \in F_{\varepsilon},|\lambda| \rightarrow \infty$.

Proof. Contrary to the assertion, we assume there exist sequences $\left(x_{n}\right) \subset \mathscr{H}_{1}$ with $\left\|x_{n}\right\|=1,\left(\lambda_{n}\right) \subset F_{\varepsilon}$ with $\left|\lambda_{n}\right| \rightarrow \infty$, and $\left(f_{n}\right) \subset \mathscr{H}$ with $0<c \leqq\left\|f_{n}\right\|$, such that $C\left(\lambda_{n}\right) x_{n}=f_{n}$. Since, by (ii), $\left(I-T\left(I-\lambda_{n} H\right)^{-1}\right)^{-1}$ is bounded on $F_{\varepsilon, R}$, we infer that $c \leqq\left\|f_{n}\right\| \leqq C$ for some $C$. Now

$$
x_{n}=\left(I-T\left(I-\lambda_{n} H\right)^{-1}\right)\left(x_{n}+f_{n}\right)
$$

and, decomposing $v \in \mathscr{H}=\mathscr{H}_{1} \oplus \mathscr{H}_{2}$ as $v=v^{1}+v^{2}$,

$$
0=f_{n}-T\left(I-\lambda_{n} H\right)^{-1} f_{n}^{2}-T\left(I-\lambda_{n} H\right)^{-1}\left(x_{n}+f_{n}^{1}\right) .
$$

The last term in (3) tends to 0 by Lemma 1 . Since $T\left(I-\lambda_{n} H\right)^{-1} f_{n}^{2}=T f_{n}^{2}$, we thus have

$$
f_{n}^{1}+(I-T) f_{n}^{2} \rightarrow 0 \quad(n \rightarrow \infty) .
$$


As $T$ is compact, we conclude that (for a subsequence) $f_{n}^{1} \rightarrow f^{1}, f_{n}^{2} \rightarrow f^{2}$, with $f=f^{1}+f^{2} \neq 0$ and $f^{1}+(I-T) f^{2}=0$. This contradicts hypothesis $\left(^{*}\right)$.

Now we note that the trace $\operatorname{sp}\left(A(\lambda)^{p}\right)=\operatorname{sp}\left(\left.A(\lambda)^{p}\right|_{\mathscr{H}_{1}}\right)$. Thus all the remaining steps of the proof of [3, Theorem 11.1, pp. 278-283] carry through unchanged. This proves Theorem 2.

\title{
References
}

1. S. Agmon, Lectures on elliptic boundary value problems, Van Nostrand Math. Studies 2 (Van Nostrand, 1965).

2. J. FLECKINGER and M. L. LAPIDUs, 'Eigenvalues of elliptic boundary value problems with an indefinite weight function', preprint.

3. I. C. GoHBERG and M. G. KREIN, Introduction to the theory of linear nonselfadjoint operators, AMS Translations of Math. Monographs 18 (Amer. Math. Soc., Providence, R.I., 1969).

4. P. Hess, 'On the relative completeness of the generalized eigenvectors of elliptic eigenvalue problems with indefinite weight functions', Math. Ann. 270 (1985) 467-475.

5. M. V. KeLDYŠ, 'On the characteristic values and characteristic functions of certain classes of nonselfadjoint equations' (Russian), Dokl. Akad. Nauk SSSR 77 (1951) 11-14.

6. Å. Pleisel, 'Sur la distribution des valeurs propres de problèmes régis par l'equation $\Delta u+\lambda k(x, y) u=0$ ', Arkiv för Mat. Astr. och Fysik 29 B (1942) 1-8.

7. A. G. Ramm 'Perturbations preserving asymptotic of spectrum', J. Math. Anal. Appl. 76 (1980) 10-17.

\author{
Mathematisches Institut \\ Universität Zurich \\ Switzerland
}

